\title{
NANO E MICROPLÁSTICOS NOS ECOSSISTEMAS: IMPACTOS AMBIENTAIS E EFEITOS SOBRE OS ORGANISMOS
}

Danila Soares Caixeta1, Frederico César Caixeta², Frederico Carlos Martins de Menezes Filho ${ }^{3}$

1 Professora Doutora do Departamento de Engenharia Sanitária e Ambiental da Universidade Federal de Mato Grosso (danilacaixeta@gmail.com) Cuiabá-Brasil

2 Graduando em Medicina pela Universidade de Cuiabá

3 Professor Doutor do Instituto de Ciências Exatas e Tecnológicas da Universidade

Federal de Viçosa - Campus Rio Paranaíba

Recebido em: 06/04/2018 - Aprovado em: 10/06/2018 - Publicado em: 20/06/2018

DOI: 10.18677/EnciBio_2018A92

\begin{abstract}
RESUMO
Os plásticos são lançados no ambiente de diferentes maneiras, podendo ser degradados por fatores abióticos e bióticos. Entretanto, quando degradadas, as partículas de dimensões na escala micro e nanoplásticas, podem se acumular no ambiente, gerando uma série de fatores hostis à flora e fauna, principalmente nos organismos. Dessa maneira, esse artigo tem como objetivo fornecer informações relevantes sobre as possíveis fontes de inserção de partículas plásticas no ambiente, bem como potencial efeito sobre os organismos. Para tal, foi realizado uma busca de artigos científicos na área da pesquisa, cadastrados em bases de dados, da comunidade científica. Nota-se que nas últimas décadas houve uma acentuada expansão de pesquisas relacionadas aos efeitos dos nano e microplásticos sobre os organismos vivos, pois esses polímeros quando presentes no ambiente podem afetar direta ou indiretamente à saúde, ecologia e economia. As consequências à saúde são em decorrência da adsorção, que posteriormente pode causar efeitos tóxicos letais aos indivíduos. Dada a crescente necessidade de detecção de nano e microplásticos no ambiente, isso se torna um grande desafio, pois, embora muitos estudos utilizem técnicas amplamente semelhantes, ainda não existe um protocolo padrão. Diante desse impasse, muitos estudos estão identificando novos critérios e relatos mais consistentes. Em suma, estudos relativos a nano e microplásticos ainda são incipientes, necessitando de mais pesquisas para preencher as lacunas existentes.
\end{abstract}

PALAVRAS-CHAVE: Polímeros, resíduos, saúde.

\section{NANO AND MICROPLASTICS IN ECOSYSTEMS: ENVIRONMENTAL IMPACTS AND EFFECTS ON ORGANISMS}

\section{ABSTRACT}


Plastics are released into the environment in different ways, and can be degraded by abiotic and biotic factors. However, when degraded, particles of micro and nanoplastic size can accumulate in the environment, generating a series of factors hostile to flora and fauna, especially in organisms. In this way, this article aims to provide relevant information about the possible sources of plastic particles in the environment, as well as potential effect on organisms. For that, a search of scientific articles in the area of research, registered in databases, of the scientific community was carried out. It is noteworthy that in the last decades there has been a sharp expansion of research related to the effects of nano and microplastics on living organisms, since these polymers when present in the environment can directly or indirectly affect health, ecology and economics. The health consequences are due to adsorption, which can later cause lethal toxic effects to individuals. Given the growing need for detection of nano and microplastics in the environment, this becomes a major challenge because, although many studies use widely similar techniques, there is still no standard protocol. Faced with this impasse, many studies are identifying new criteria and more consistent reports. In summary, studies on nano and microplastics are still incipient, requiring further research to fill existing gaps.

KEYWORDS: Health, polymers, waste.

\section{INTRODUÇÃO}

Nas últimas décadas a produção de plástico tem aumentado significantemente, devido a aplicação em vários produtos, de diferentes setores. Dentre os polímeros mais comuns, destaca-se o polipropileno (PP), polietileno (PE), policloreto de vinila (PVC), poliestireno (PS) e polietileno tereftalato (PET), que correspondem a 90\% da demanda de plástico no mundo (ANDRADY; NEAL, 2009). Contudo, a demanda por esses materiais é em função do seu baixo custo, alta durabilidade e, também, por sua resistência à radiação, produtos químicos, pressão, dentre outros.

Em consequência do consumo de produtos manufaturados por esses polímeros, são geradas grandes quantidades de resíduos, que por sua vez, nem sempre são reciclados ou reutilizados, sendo então, lançados de forma direta ou indireta no ambiente, causando uma série de danos. Desse modo, as atividades antrópicas e industriais são consideradas altamente impactantes, pois são as principais fontes de inserção de plásticos no ambiente. Segundo Carvalho e Baptista Neto (2016) os materiais plásticos correspondem de 60 a $80 \%$ dos detritos encontrados nos oceanos, provenientes das atividades antrópicas.

Os plásticos podem ser degradados por fatores abióticos ou bióticos, sendo a radiação UV, a ação mecânica e a hidrólise, de fundamental importância na fragmentação e disponibilização dos mesmos, aos microrganismos. Assim, o processo de degradação, gera partículas em escalas, meso, micro e nano, os quais são classificados em mesoplásticos, microplásticos e nanoplásticos, respectivamente (COSTA et al., 2016).

O termo microplástico foi primeiramente mencionado por Thompson et al. (2004) caracterizando partículas com tamanho de $20 \mu \mathrm{m}$. No entanto, até o presente momento, não existe um consenso entre os autores, em relação à padronização do tamanho das partículas.

Os microplásticos e os nanoplásticos são os polímeros de maior impacto no ambiente, pois devido às suas extensas áreas de superfície podem adsorver 
compostos altamente tóxicos, tais como hidrocarbonetos e metais pesados. Inquestionavelmente, quando disponíveis, essas partículas podem ser adsorvidas pelos organismos e atravessar as barreiras imunológicas, afetando órgãos, tecidos e até mesmo a funcionalidade da célula, ocasionando ainda efeitos tóxicos ou letais (RAFIEE et al., 2018). Nesse aspecto, vários estudos apontam em seus resultados, os danos causados em crustáceos, invertebrados, peixes, algas e zooplâncton (MA et al., 2016; CHAE; AN, 2017; BERGAMI et al., 2017). Entretanto, em humanos as pesquisas ainda são incipientes, necessitando de mais alicerce científico.

Em humanos, as principais causas de contaminação por micro e nanoplásticos ocorrem através das vias aéreas, contato com produtos de uso pessoal, consumo de alimentos e água, provocando danos diretos ou indiretos na homeostase do organismo (HORTON et al., 2017; PRATA, 2018). Em síntese, os impactos ocasionados pelos plásticos afetam todo o ecossistema.

Embora sejam encontrados nos mais diversos ambientes, água, ar, solo, biofilme, superfície de animais, e outros, a detecção e identificação de microplásticos e nanoplásticos é um desafio visto que, ainda, não foi estabelecida uma metodologia padrão. Dessa forma, recomenda-se que seja utilizada mais de uma técnica por amostragem, a fim de fornecer resultados qualitativos e quantitativos, confiáveis. Diante disso, pesquisadores de diferentes áreas estão empenhados a estabelecer a melhor técnica para detecção e identificação de plásticos em amostras ambientais.

Esse artigo de revisão teve como objetivo fornecer informações relevantes sobre a possíveis fontes de inserção de partículas plásticas no ambiente, bem como potencial efeito sobre os organismos.

\section{DESENVOLVIMENTO}

\section{Plásticos: produção e expansão comercial}

O termo plástico é usado genericamente para designar material polimérico sintético e semi-sintético, comumente derivado de petróleo, e que exibe alta massa molecular e plasticidade (COSTA et al., 2016). Composto por componentes orgânicos, possuem unidades químicas ligadas covalentemente, repetidas regularmente ao longo da cadeia, denominadas meros, sendo o número de meros da cadeia polimérica denominado grau de polimerização, que podem ser moldados por ação de calor e/ou pressão (MANO; MENDES, 1999; COSTA, 2018).

A produção e comercialização dos plásticos iniciou-se no século XIX e no século XX ocorreu uma significativa expansão. Entre 1950 e 2016, a produção passou de 1,7 para 322 milhões de toneladas, sendo a Europa, produtora de 57 milhões de toneladas no ano de 2012 (PLASTIC EUROPE, 2017; REVEL et al., 2018). Dados de 2016 da Plastics Europe Market Research Group (PEMRG) e Conversion Market \& Strategy $\mathrm{GmbH}$, mostram os principais setores, em que há maior demanda de plásticos na Europa, tendo em vista que as embalagens correspondem ao maior percentual (39,9\%), seguido de construções $(19,7 \%)$, indústria automotiva $(10 \%)$, indústria elétrica e eletrônicos $(6,2 \%)$, produtos de uso pessoal $(4,2 \%)$, agricultura (3,3\%) e outros (16,7\%) (PLASTICS EUROPE, 2017). Indubitavelmente, tais aplicações em uma ampla variedade de produtos estão relacionadas a facilidade de fabricação, baixo custo, resistência à água, a produtos químicos, temperatura e à luz. 
Como consequência dos usos múltiplos dos plásticos, grandes impactos ao ambiente são gerados, uma vez que a destinação dos produtos manufaturados a partir desses polímeros, são lançados indiscriminadamente. Todavia, medidas de preservação ambiental estão sendo adotadas, sendo a prática de reciclagem a mais comumente utilizada. Neste enfoque, Barros (2014) ressalta a importância da reciclagem de plásticos, pela economia de matéria-prima e energia para a fabricação de novos materiais, sendo que a destinação final dos mesmos em aterros sanitários configura um desperdício de tais recursos naturais.

$\mathrm{Na}$ Europa, no ano de 2016, por meio de intervenções oficiais, foram coletados 27,1 milhões de toneladas de resíduos plásticos, para serem tratados e pela primeira vez, mais resíduos de plástico foram reciclados do que aterrados. Estima-se que destes $31,1 \%$ foram reciclados, $43,6 \%$ foram utilizados na produção de energia e apenas 27,3\% foram depositados em aterros (PLASTICS EUROPE, 2017). No Brasil, cerca de $21,7 \%$ dos plásticos foram reciclados em 2011, representando aproximadamente 953 mil toneladas por ano (CEMPRE, 2018).

Visto que uma grande parcela de plástico, ainda não é reciclada e/ou reaproveitada, este é lançado de forma inapropriada no ambiente, tornando-se recalcitrante ou susceptíveis à degradação por processos bióticos e abióticos. Os processos abióticos, dentre eles a degradação mecânica, química, fotodegradação e térmica são de grande relevância, pois são capazes de promover perdas de propriedades estruturais e mecânicas, aumentando a área de superfície disponível para a colonização microbiana (COSTA et al., 2016).

Ademais, a degradação biótica ou biodegradação é um processo enzimático mediado por microrganismos, que são capazes de decompor materiais em dióxido de carbono, metano, água, compostos inorgânicos e biomassa. Porém, salienta-se que tal processo pode ser influenciado pelas características do polímero, propriedades físicas e químicas, bem como por fatores ambientais, tais como luz (UV), calor, umidade e presença de agentes químicos (PAÇO et al., 2017).

Cai et al. (2018), realizaram estudo com o objetivo de verificar a degradação de três diferentes plásticos por meio da radiação. No entanto, os resultados revelaram que não houve diferença significativa na resistência dos pellets plásticos, mas houve grande diferença nas características após exposição à radiação, utilizando-se como meio de detecção o Espectro de Raman.

\section{Dimensões de partículas plásticas}

Dada a importância da universalização das dimensões dessas partículas, pesquisadores de várias áreas têm buscado instrumentos para padronizar a classificação destas, todavia sem consenso conforme mostrado na Tabela 1.

Tabela 1 Classificações de plásticos e suas dimensões.

\begin{tabular}{|c|c|c|c|c|}
\hline & $\begin{array}{l}\text { Nanoplástic } \\
0\end{array}$ & $\begin{array}{l}\text { Microplástic } \\
\text { o }\end{array}$ & Mesoplástico & $\begin{array}{l}\text { Macroplástic } \\
\text { o }\end{array}$ \\
\hline $\begin{array}{l}\text { GALGANI et al., } \\
2013\end{array}$ & - & $25 \mu \mathrm{m}-5 \mathrm{~mm}$ & $5-2,5 \mathrm{~cm}$ & - \\
\hline $\begin{array}{l}\text { WAGNER et al., } \\
2014\end{array}$ & $<20 \mu \mathrm{m}$ & - & - & - \\
\hline $\begin{array}{l}\text { KOELMANS et } \\
\text { al., } 2015\end{array}$ & $<100 \mathrm{~nm}$ & - & - & - \\
\hline COSTA et al., & $<1 \mu \mathrm{m}$ & $=$ & - & - \\
\hline
\end{tabular}


Nos anos de 1971 e 1972, Buchanan, Carpenter e Smith observaram pela primeira vez partículas de plásticos no ambiente marinho Buchanan (1971) e Carpenter e Smith (1972), no entanto foi somente em 2004 que o termo microplástico se tornou comum, devido a um estudo realizado por Thompson et al. (2004). Nesse estudo, Thompson et al. (2004) objetivaram quantificar a abundância de microplásticos em amostras de sedimentos de praias; e sedimento e estuário de Plymouth, UK, sendo identificados 9 polímeros.

Lambert e Wagner (2016) definiram microplásticos como sendo partículas com dimensões menores que $5 \mathrm{~mm}$, enquanto que Gigault et al. (2018) propuseram o termo nanoplástico para definir pequenos plásticos, tais como partículas simples ou em forma de homo e/ou heteroagregados, com dimensões entre $1 \mathrm{~nm}$ a $1 \mu \mathrm{m}$.

De acordo com Horton et al. (2017) os microplásticos são fundamentalmente obtidos a partir de duas fontes: primária que são os plásticos virgens (pellets), produzidos especificamente em escala micrométrica; e a secundária que são os fragmentos resultantes da degradação física, química e biológica de meso e macroplásticos (HATJE et al., 2013). Li et al. (2017) afirmam que os microplásticos secundários são a maioria, e sua multiplicação no ecossistema é em função da entrada de detritos plásticos de diferentes origens.

Tendo em vista isso, os nanoplásticos e microplásticos são provenientes da quebras de grandes detritos plásticos, devido a exposição a atividade de animais e microrganismos, bem como degradação por processos naturais.

\section{Plásticos e suas fontes}

Como consequência do uso em diversos setores, bem como da resistência a tratamentos químicos, térmicos e a degradação, os plásticos podem ser encontrados nos mais variados ambientes, tais como solo, água, ar, lodo de esgoto, águas tratadas, sedimento e outros (DEKIFF et al., 2014; CARR et al., 2016; CARVALHO; BAPTISTA NETO, 2016).

Em ambientes terrestres, as principais vias de acesso de microplásticos são as resultantes de práticas agrícolas, tais como a aplicação de lodo de esgoto contendo fibras sintéticas ou microplásticos sedimentados, bem como os provenientes de cuidados pessoais ou produtos domésticos; deposição por escoamento e fragmentação de plásticos de dimensões maiores (HURLEY; NIZZETTO, 2017; HORTON et al., 2017).

Além disso, em ambientes aquáticos as fontes de inserção de partículas, de dimensões nano e microplásticas são: (1) as produzidas para propostas específicas, tais como produtos cosméticos, tintas de impressoras, produção de fármacos; (2) fragmentação de plásticos devido à fotodegração por UV, ação mecânica, hidrólise e atividade microbiana; e (3) em sistemas de tratamento de água (biossólidos e efluente líquido) (COSTA et al., 2016; MENDOZA et al., 2018). Lambert e Wagner (2016) corroboram com dados da literatura, comprovando que os nanoplásticos podem ter sua origem a partir da degradação por radiação UV. 
Estahbanati e Fahrenfeld (2016) investigaram a concentração de microplásticos no rio de água doce (Raritan) localizado em Nova Jersey, sendo as amostras coletadas a montante e a jusante de quatro pontos de lançamento de efluente. Os resultados evidenciaram uma maior concentração de microplásticos a jusante dos pontos de lançamento, com tamanho variando entre 125 a $500 \mu \mathrm{m}$.

Verifica-se ainda, que em sistemas aquáticos, o lixo produzido por atividades de recreação e pesca, também são importantes fontes. Em ambiente marinho, estudos mostraram que entre 70 a $80 \%$ dos microplásticos existentes nas águas oceânicas, são provenientes de rios, os quais são resultantes de atividades antrópicas (HORTON et al., 2017).

De modo geral, as principais fontes de poluição incluem as de origem industrial, como as matérias-primas utilizadas na fabricação de produtos plásticos e de derramamento de pós ou pellets de resina plástica usados para a remoção de ar. Atividades industriais, como a corte térmico de espuma de poliestireno, demonstrou emitir partículas de polímero de tamanho nanométrico, na faixa de aproximadamente 20-220 nm (COSTA et al., 2016). Além disso, essas partículas podem ser transferidas para o ambiente, através da desintegração de folhas de polietileno agrícola $(\mathrm{PE})$, lavagem de roupas sintéticas e lodo de esgoto contaminado, empregado como fertilizante. Segundo Mendoza et al. (2018) as partículas de nanoplásticos de maior relevância, referem-se às fibras sintéticas, sendo a lavagem de roupas, provedora de aproximadamente 1900 fibras por cada peça lavada.

Por outro lado, a presença de nano e microplásticos em águas tratadas, são provenientes de produtos de limpeza e cosméticos que são lançados em águas residuárias doméstica, e estes nem sempre são removidos por técnicas convencionais (COSTA et al., 2016).

Schymanski et al. (2018) verificaram a presença de microplásticos em amostras de água potável, armazenadas em garrafas plásticas, garrafas de vidro e caixas de bebidas, com partícula de dimensões variando entre 5 e $20 \mu \mathrm{m}$, sendo os tipos de polímeros predominantes o polietileno tereftalato (PET), seguidos de polietileno (PE), poliestireno (PS) e polipropileno (PP). Por outro lado Imhof et al. (2016), verificaram a alta incidência de microplásticos em sedimentos obtidos do Lago de Garda na Itália, com tamanhos que variaram entre 50-500 $\mu \mathrm{m}$.

\section{Efeitos dos plásticos no ambiente}

Os nanoplásticos e os microplásticos, por apresentarem alta área de superfície, podem promover uma forte sorção de compostos tóxicos, como hidrocarbonetos policíclicos aromáticos (PAHs), bifenilos policlorados (PCBs) e dicloro difenil tricloroetano (DDTs) (BERGAMI et al., 2017). Entretanto, o tamanho e a textura das partículas podem afetar a capacidade de adsorver ou lixiviar contaminantes, enquanto que as condições ambientais podem influenciar na dinâmica de equilíbrio entre produtos químicos e plásticos, impactando na acumulação química e na biodisponibilidade (HORTON et al., 2017).

Assim, quando disponível no ambiente, nano e microplásticos, podem interagir com os organismos através da ingestão, atravessando as barreiras biológicas, penetrando e acumulando nos tecidos e órgãos. Como consequência da ingestão de tais partículas citam-se os efeitos toxicológicos, devido à permanência de poluentes orgânicos persistentes, tais como hidrocarbonetos policíclicos aromáticos, éteres difenílicos polibromados e bifenilos policlorados, além de outros efeitos adversos, como a inibição de crescimento, desordens comportamentais e 
alimentares, disfunção reprodutiva, viabilidade reduzida, mobilidade e até mesmo a morte (MA et al., 2016; CHAE; AN, 2017; COSTA, 2018).

De acordo com Mendoza et al. (2018) os microplásticos são os polímeros sintéticos, mais persistentes em ambientes aquáticos, causando efeitos ecotoxicológicas e por vezes deletérias em organismos aquáticos. Outrossim, os nanoplásticos têm a capacidade de atravessar a membrana citoplasmática e alterar a funcionalidade das células, incluindo células sanguíneas e até mesmo o processo fotossintético (COSTA et al., 2016).

De modo geral, esses contaminantes quando presentes em ambientes diversos, por consequência podem desequilibrar a cadeia alimentar dos seres vivos e por conseguinte afetar a saúde humana. Segundo Carbery et al. (2018) as evidências para a transferência trófica de microplásticos, surgiu a partir da quantificação desses polímeros em organismos coletados em campo, seus predadores naturais e estudos que simulam a transferência através da cadeia alimentar. Cedervall et al. (2012) mostraram que nanopartículas de poliestireno fabricadas comercialmente, são transportados através da cadeia alimentar aquática de algas, que posteriormente são ingeridos pelos zooplâncton e finalmente por peixes, afetando por conseguinte seu comportamento e metabolismo lipídico.

Por outro lado, os efeitos tóxicos dos nano e microplásticos, vêm sendo estudados desde a década de 80 , caracterizando seus danos letais e subletais em algas, organismos ciliados, invertebrados, crustáceos, peixes e zooplânctons (CHAE; AN, 2017). Prata (2018) menciona que microplásticos têm mostrado reduzir o processo fotossíntetico e crescimento de microalgas, provocado efeitos negativos na cadeia alimentar de zooplâncton, que acumula e possivelmente provoca sequelas em brânquias, estômago e hepatopâncreas de caranguejos e induz alterações nos tecidos e biomarcadores de peixes. Estudo realizado por Sjollema et al. (2016) corroba que microplásticos afetam os processos fotossíntéticos e crescimento de microalgas.

Resultados obtidos por Ma et al. (2016) evidenciaram que as partículas de plásticos com dimensões de $50 \mathrm{~nm}$ apresentaram toxicidade e causou danos físicos a Daphnia magna. Os resultados ainda mostraram que a presença de partículas de plástico aumentou significantemente a biocumulação de resíduos derivados de fenantreno no corpo de Daphnia magna, diminuindo a dissipação e transformação do fenantreno no meio.

Bergami et al. (2017) avaliaram o efeito de dois nanoplásticos de poliestireno com superfície modificada (40 nm PS-COOH and $50 \mathrm{~nm}$ PS- $-\mathrm{NH}_{2}$ ) sobre espécies planctônicas de Dunaliella tertiolecta e do crustáceo Artemia franciscana. No entanto, os autores, observaram um comportamento diferente das duas superfícies modificadas, em meios de exposição, bem como toxicidade diversa para as duas espécies, onde o nanoplástico de poliestireno com superfície modificada (PS-COOH) formou agregados em micro escala, porém nao afetou o crescimento das microalgas até $50 \mu \mathrm{g} / \mathrm{ml}$ e de camarões de salmoura até $10 \mu \mathrm{g} / \mathrm{ml}$.Todavia, esses naonoplásticos carregados negativamente foram adsorvidos em microalgas e acumulados em camarões de salmoura, sugerindo uma transferência trófica potencial de presa para predador.

Embora várias pesquisas comprovem os efeitos tóxicos sobre os organismos, estudos eventuais sobre os impactos tóxicos dos nano e microplásticos na saúde humana têm sido limitados (REVEL et al., 2018). No entanto, alguns estudos mostram que os nanoplásticos e microplásticos têm potencial para afetar direta ou indiretamente a saúde. 
Relatos recentes mostraram que o consumo de animais contaminados; água tratada com partículas plásticas; adsorção pela pele quando em contato com cosméticos que apresentam grande concentração de nanoplásticos; inalação do ar contendo partículas provenientes de sistema de tratamento de água e exposição de lodo no solo, dentre outras, podem eventualmente acometer a saúde humana, com implicações no intestino e outros órgaos, bem como ocasionar efeitos tóxicos, devido a habilidade de induzir bloqueio intestinal e/ou tecidual, além de fibroses, congestão e inflamações (REVEL et al., 2018).

Schirinzi et al. (2017) objetivaram em seus estudos verificar os efeitos de nanopartículas de prata (AgNPs), nanopartículas de ouro (AuNPs), nanopartículas de dióxido de zircónio ( $\left.\mathrm{ZrO}_{2} \mathrm{NPs}\right)$, nanopartículas de óxido de cério $\left(\mathrm{CeO}_{2} \mathrm{NPs}\right)$, nanopartículas de dióxido de titânio ( $\left.\mathrm{TiO}_{2} \mathrm{NPs}\right)$, nanopartículas de óxido de alumínio $\left(\mathrm{Al}_{2} \mathrm{O}_{3} \mathrm{NPs}\right.$ ), $\mathrm{C} 60$ fulereno, grafeno e microplásticos (polietileno e poliestireno) em nível celular em termos de estresse oxidativo e viabilidade celular, sendo utilizados duas linhas de células diferentes T98G e HeLa, células humanas cerebrais e epiteliais, respectivamente. Os resultados mostraram que nenhum dos nanomateriais e microplásticos, estudados, conduziram a uma redução significativa da viabilidade celular, mas em contra partida, os resultados confirmam que o estresse oxidativo é um dos mecanismos de citotoxicidade no nível celular, como observado para ambas as linhas celulares. Ademais, estudo realizado por Revel et al. (2018) mostra que ratos quando expostos a microplásticos, induz o estresse oxidativo, altera o metabolismo energético e lipídico e apresenta efeitos neurotóxicos.

Em síntese, a parcela lançada no ambiente, principalmente os plásticos de dimensões nano e micro, tem causado grandes prejuízos ao meio ambiente, à ecologia, à economia e à saúde pública. Complementarmente, devido a falta de informação sobre a toxicologia dos nanoplásticos, restringe-se o uso deles para certas aplicações, que estão diretamente em contato com seres humanos, como a inclusão em cosméticos, detergentes e alimentos, a fim de prevenir seu potencial de toxicidade e efeitos adversos secundários de longo prazo (SCHIRINZI et al., 2017).

\section{Tecnologias de detecção de plásticos no ambiente}

Devido às limitações metodológicas, atualmente, poucos estudos têm detectado microplásticos em amostras ambientais com dimensão de $1 \mu \mathrm{m}$. Assim, o ponto de partida para análises de plásticos são baseados nas dimensões do mesmos, por conseguinte, é de extrema importância que os pesquisadores padronizem os tamanhos das partículas, bem como as unidades de medidas, a fim de relatar as concentrações nano e microplásticos. Para comparar estudos onde as unidades não são consistentes, as unidades devem ser transformadas em unidades por volume, seja como partículas por litro de água amostrada ou como partículas por quilograma de sedimento ou solo (HORTON et al., 2017).

O Guidance on Monitoring of Marine Litter in European Seas publicado em 2013 pela Comissão Européia é um guia de orientação amplo, que objetiva direcionar os pesquisadores em relação ao monitoramento, adequação de metodologias de monitoramento, custos e outros parâmetros relativos ao ambiente marinho, dando subsídios a toma de decisões, sobre a melhor maneira de conduzir os estudos (EUROPEAN COMMISSION, 2013).

De fato, pode ser verificado que a grande maioria das pesquisas relatadas na literatura, utilizam mais de uma metodologia para identificação de nano e 
microplásticos, nos mais variados segmentos, tais como água, sedimentos, organismos alimentos e outros. A abordagem mais comumente aplicada para a detecção de nano e microplásticos consiste na identificação visual, que pode ser realizada apenas por observação a olho nu ou com auxílio de um microscópio óptico. Esta apresenta uma boa resposta, quando utilizada para analisar polímeros com dimensões de aproximadamente $500 \mu \mathrm{m}$, entretanto a sua aplicação gera controvérsias entre os pesquisadores, devido aos erros humanos que podem comprometer a veracidade das informações. Assim sua confirmação deverá ser seguida de análises de composição química, geralmente combinando técnicas ópticas e espectroscópicas, a fim de minimizar a ocorrência de falso positivo e/ou falsos negativos (SILVA et al., 2018).

Algumas pesquisas abordam que, em função dos diferentes tamanhos das partículas, essas devem passar por um pré tratamento, ou seja, serem submetidas à separação por densidade, que pode ser pela aplicação de $\mathrm{KOH}$ ou $\mathrm{NaCl}$. Dessa forma, o pré-tratamento tem como finalidade auxiliar na extração, purificação, e conservação da estrutura dos plásticos, além de remover partículas que não são plásticos, tais como biofilmes, sais, sangue, entre outros (QIU et al., 2016). Por outro lado, alguns autores não veem coerência na aplicação do pré-tratamento (RENNER et al., 2018), uma vez que ácidos oxidantes como $\mathrm{H}_{2} \mathrm{SO}_{4}, \mathrm{HNO}_{3}$, podem destruir alguns tipos de plásticos que apresentam baixa tolerância ao pH, como exemplo o poliestireno e nylon, enquanto que substâncias alcalinas podem danificar e descolorir nylon e polietileno (QIU et al. 2016).

Dada a crescente necessidade de fazer avaliações comparativas para identificar tendências regionais, nacionais e globais na distribuição de nano e microplásticos no ambiente, isso se torna um grande desafio, pois, embora muitos estudos utilizem técnicas amplamente semelhantes, ainda não existe um protocolo padrão. Diante desse impasse, muitos estudos estão identificando novos critérios e relatos mais consistentes.

Recentemente, houve um aumento do uso de FTIR (Espectroscospia de Infravermelho), por possibilitar a uma detecção de partículas com tamanhos inferior a $500 \mu \mathrm{m}$, o Espectroscópio de Raman (partículas $\leq 20 \mu \mathrm{m}$ ), Microscopia de Fluorescência, Microscopia Eletrônica de Varredura, Microscopia de Força Atômica, Cromatografia Gasosa com Espectrômetro de Massa, dentre outros.

A disposição de nanoplásticos (PS-COOH) em micro alga verde foi observado por Microscópio de Fluorescência, enquanto a disposição de nanoplástico (PS-NH $\mathrm{NH}_{2}$ foi determinado usando o Microscópio Eletrônico de Varredura (BERGAMl et al., 2017). Em adição Imhof et al. (2016) verificaram a incidência de pigmentos e plásticos em ecossistemas linminéticos por meio de Microespectroscópio de Raman. Enquanto que Erikesen et al. (2013) detectaram vários tipos de plásticos no Lago de Laurentian Great, utilizando a técnica de separação visual e Microscopia Eletrônica de Varredura.

Schymanski et al. (2018) mencionam que as técnicas espectroscopia de infravermelho de micro-transformação de Fourier ( $\mu$-FT-IR), mais comumente utilizadas para investigações de microplásticos em água potável apresentam limitações, pois não tem capacidade de detectar partículas menores que $20 \mu \mathrm{m}$, mas que a Microespectroscópio de Raman é mais eficiente, por ser capaz de detectar tamanhos de partículas ainda menores.

\section{Instrumentos legais de lançamento de nano e microplásticos no ambiente}


Com a indiscutível expansão da produção de plásticos em todo o mundo, os impactos gerados abrangem as esferas ambiental, social e econômica. Neste enfoque, a não padronização de metodologias para inspeção dos impactos dificultam a confiabilidade dos resultados tão quanto a ausência de instrumentos legais quantificando limites aceitáveis de nano e microplásticos. Admite-se, assim, que as legislações brasileiras, em vigor, efetivamente não dispõem sobre os valores máximos toleráveis, que sejam seguros à saúde.

Na tabela 2 apresentam-se apenas os valores de referência para monômeros de cloreto de vinila (PVC) e poliestireno (estireno).

Tabela 2 Valores de referência para monômeros de cloreto de vinila e poliestireno, em amostras de água, solo e alimentos

\begin{tabular}{|c|c|c|}
\hline & Cloreto de Vinila & Estireno \\
\hline $\begin{array}{l}\text { Portaria No } 2.914 / 2011 \\
\text { (Água potável) (MS, 2011) }\end{array}$ & $5 \mu \mathrm{g} / \mathrm{L}$ & $20 \mu \mathrm{g} / \mathrm{L}$ \\
\hline $\begin{array}{l}\text { Resolução No } 430 / 2011 \\
\text { (Efluente) (CONAMA, 2011) }\end{array}$ & & $0,07 \mathrm{mg} / \mathrm{L}$ \\
\hline $\begin{array}{l}\text { Resolução No } 420 / 2009 \\
\text { (Águas } \quad \text { subterrâneas) } \\
\text { (CONAMA, 2009) }\end{array}$ & $5 \mu \mathrm{g} / \mathrm{L}$ & $20 \mu \mathrm{g} / \mathrm{L}$ \\
\hline $\begin{array}{l}\text { Resolução RDC No } 274 / 2005 \\
\text { (Águas envasadas e gelo) } \\
\text { (ANVISA, 2005) }\end{array}$ & $5 \mu \mathrm{g} / \mathrm{L}$ & $20 \mu \mathrm{g} / \mathrm{L}$ \\
\hline $\begin{array}{l}\text { Resolução } N^{\circ} 420 / 2009 \\
\text { (Solo residencial) (CONAMA, } \\
2009)\end{array}$ & $\begin{array}{c}0,003 \mathrm{mg} / \mathrm{Kg} \text { peso seco } \\
\text { solo }\end{array}$ & $\begin{array}{l}35 \mathrm{mg} / \mathrm{Kg} \text { peso } \\
\text { seco solo }\end{array}$ \\
\hline
\end{tabular}

Em aproximadamente 40 países, incluindo Estados Unidos, Canadá, França Itália, dentre outros, existem organizações e comitês, que buscam instrumentos regulatórios, para minimizar os impactos gerados pelos plásticos em ambientes marinhos, incluindo planos de ação, estratégias, regulamentação, diretrizes, e acordos que objetivam prevenir, remover, mitigar e educar (COSTA, 2018). Ademais, Organizações Não-Governamentais (ONGs) também criaram programas que visam conscientizar e ajudar a quantificar a extensão da poluição por microplásticos e os efeitos em escala nacional, regional e internacional (JIANG, 2018).

A America's Plastics Makers trabalha em sintonia com cientistas e formuladores de políticas, que tem como objetivo descobrir soluções eficazes da destinação de lixo marinho, a fim de promover ações para aumentar a prevenção, redução, reutilização e reciclagem de lixo, e outras infraestruturas de gestão de resíduos, juntamente com parcerias regionais e internacionais (MARINE LITTER SOLUTIONS, 2018).

Contudo, a aplicação das normas regulatórias e legislações existentes, nesses países, podem contribuir na redução e prevalência de plásticos e subsequente micro e nanoplásticos no ambiente. Como consequência, irá promover melhor qualidade de vida da população existente nesses ecossistemas, assim como estabelecer um equilíbrio ecológico. 


\section{CONCLUSÃO}

A demanda por materiais manufaturados a partir de plásticos, tem crescido consideravelmente nas últimas décadas. Todavia, esta expansão é acompanhada com a geração de toneladas de resíduos, alguns dos quais são subprodutos não biodegradáveis e tóxicos, resultando na acumulação no ambiente.

Uma vez presente no ambiente, esses polímeros podem afetar direta ou indiretamente a saúde, ecologia e economia. Vários relatos mostram que os efeitos nocivos à saúde podem ser desde a adsorção, até mesmo causar efeitos tóxicos letais, podendo ainda, acometer a saúde de humanos através da cadeia alimentar.

No entanto, devido as várias limitações metodológicas na quantificação e detecção de nano e microplásticos no ambiente, bem como em animais e humanos, estudos ainda são incipientes, necessitando de mais pesquisas para poder preencher as lacunas existentes, principalmente no que tange aos efeitos a longo e curto prazo.

\section{REFERÊNCIAS}

ANDRADY, A. L.; NEAL, M. A. Applications and societal benefits of plastics. Philosophical Transactions Royal Society, v. 364, n. 1526, p. 1977-1984, 2009. Disponível em: <http://doi.org/10.1098/rstb.2008.0304>. doi:10.1098/rstb.2008.0304.

ANVISA. Resolução RDC $n^{\circ} 274$, de 22 de setembro de 2005. A prova o regulamento técnico para águas envasadas e gelo. Diário Oficial da União, Poder Executivo, Brasília, DF, 23 set. 2005.

BERGAMI, E.; PUGNALINIA, S., VANNUCCINIA, M. L.; MANFRA, L.; FALERI, C.; SAVORELLI, F.; DAWSON, K. A.; CORSIA, I. Long-term toxicity of surface-charged polystyrene nanoplastics to marine planktonic species Dunaliella tertiolecta and Artemia franciscana. Aquatic Toxicologyc, v. 189, p.159-169, 2017.Disponível em: <http://doi:10.1016/j.aquatox.2017.06.008>. doi:10.1016/j.aquatox.2017.06.008.

BARROS, R. M. Resíduos Sólidos. Ciências Ambientais para Engenharia. $1^{\text {a }}$. ed. Rio de Janeiro: Elsevier, p.157-191, 2014.

BUCHANAN, J. B. Pollution by synthetic fibres. Marine Pollution Bulletin, v. 2, n. 2, p. 23, 1971. Disponível em: <https://doi.org/10.1016/0025-326X(71)90136-6>. doi:10.1016/0025-326X(71)90136-6.

CAI, L.; WANG, J.; PENG, J.; WU, Z.; TAN, X. Observation of the degradation of three types of plastic pellets exposed to UV irradiation in three diferente environments. Science of the Total Environment, v. 628-629, p. 740-747, 2018. Disponível em: <https://doi.org/10.1016/j.scitotenv.2018.02.079 0048-9697>. Doi:10.1016/j.scitotenv.2018.02.079 0048-9697.

CARBERY, M.; O'CONNOR, W.; PALANISAMI, T. Trophic transfer of microplastics and mixed contaminants in the marine food web and implications for human health. Environment International, 2018. Disponível em: <https://doi.org/10.1016/j.envint.2018.03.007>. doi: org/10.1016/j.envint.2018.03.007. 
CARPENTER, E.J.; SMITH, K. Plastics on the Sargasso Sea surface. Science, v. 175 p. 1240-1241, 1972. Disponível em: <https://www.ncbi.nlm.nih.gov/pubmed/5061243>.

CARR, S. A.; LIU, J.; TESORO, A. G. Transport and fate of microplastic particles in wastewater treatment plants. Water Research, v. 91, p. 174-182, 2016. Disponível em: $\quad<$ http://dx.doi.org/10.1016/j.watres.2016.01.002 0043-1354>. doi:10.1016/j.watres.2016.01.002 0043-1354.

CARVALHO, D. G.; BAPTISTA NETO, J. A. Microplastic pollution of the beaches of Guanabara Bay, Southeast Brazil. Ocean \& Coastal Management, v. 128, p. 10-17, 2016. Disponível em: <http://dx.doi.org/10.1016/j.ocecoaman.2016.04.009 09645691>. doi:10.1016/j.ocecoaman.2016.04.0090964-5691.

CEDERVALL, T.; HANSSON, L. LARD, M.; FROHM, B.; LINSE, S. Food chain transport of nanoparticles affects behaviour and fat metabolism in fish. Plos One, v. 7, n. 2 2 6 p. 2012. Disponível em: http://doi.org/10.1371/journal.pone.0032254 doi:10.1371/journal.pone.0032254.

CEMPRE. Plásticos. São Paulo. 2018. Disponível em: < http://cempre.org.br/artigopublicacao/ficha-tecnica/id/4/plasticos>. Acesso: 22 fev. 2018.

CHAE, Y.; AN, Y. J. Effects of micro- and nanoplastics on aquatic ecosystems: current research trends and perspectives. Marine Pollution Bulletin, v. 124, p. 624632, 2017. Disponível em: <https:// doi:10.1016/j.marpolbul.2017.01.070>. doi: 10.1016/j.marpolbul.2017.01.070.

CONAMA. Resolução $n^{\circ} 420$, de 28 de dezembro de 2009. Dispõe sobre critérios e valores orientadores de qualidade do solo quanto à presença de substâncias químicas e estabelece diretrizes para o gerenciamento ambiental de áreas contaminadas por essas substâncias em decorrência de atividades antrópicas. Diário Oficial da União, Poder Legilativo, Brasília, DF, 30 dez. 2009.

CONAMA. Resolução CONAMA N 430/2011. Dispõe sobre condições e padrões de lançamento de efluentes, complementa e altera a Resolução no 357, de 17 de março de 2005, do Conselho Nacional do Meio Ambiente - CONAMA. Diário Oficial da União, Poder Legislativo, Brasília, DF, 16 mai. 2011.

COSTA, J. P. Micro and nanoplastics in the environment: research and policymaking. Current Opinion in Environmental Science \& Health, v. 1, p. 12-16, 2018. Disponível em: <https://doi.org/10.1016/j.coesh.2017.11.002>. doi:10.1016/j.coesh.2017.11.002.

COSTA, J. P. SANTO, P. S. M., DUARTE, A. C.; ROCHA-SANTOS, T. (Nano)plastics in the environment - Sources, fates and effects. Science of the Total Environment, v. 566-567, p. 15-26, 2016. Disponível em: $<$ https://doi.org/10.1016/j.scitotenv.2016.05.041>. doi:10.1016/j.scitotenv.2016.05.041. 
COSTA, M. F.; COSTA, J. P.; DUARTE, A. C. Sampling of micro(nano)plastics in environmental compartments: How to define standard procedures?. Current Opinion in Environmental Science \& Health, v. 1, p. 36-40, 2018. Disponível em: $<$ https://doi.org/10.1016/j.coesh.2017.10.001>. doi:10.1016/j.coesh.2017.10.001.

DEKIFF, J. H.; REMY, D.; KLASMEIER, J.; FRIES, E. Occurrence and spatial distribution of microplastics in sediments from Norderney. Environmental Pollution, v. 186, p. 248-256, 2014. Disponível em: <http://dx.doi.org/10.1016/j.envpol.2013.11.019>. doi:10.1016/j.envpol.2013.11.019.

ERIKSEN, M.; MASON, S.; WILSON, S.; BOX, C.; ZELLERS, A.; EDWARDS, W.; FARLEY, H.; AMATO, S. Microplastic pollution in the surface waters of the Laurentian Great lakes. Marine Pollution Bulletin, v. 77, p. 177-182, 2013. Disponível em: doi:10.1016/j.marpolbul.2013.10.007. $<$ http://dx.doi.org/10.1016/j.marpolbul.2013.10.007>.

ESTAHBANATI, S.; FAHRENFELD, N. L. Influence of wastewater treatment plant discharges on microplastic concentrations in surface water. Chemosphere, v. 162, p. 277-284, 2016.2 Disponível em: <https://doi.org/10.1016/j.chemosphere.2016.07.083>. doi:10.1016/j.chemosphere.2016.07.083.

EUROPEAN COMMISSION. Guidance on Monitoring of Marine Litter in European Seas. European Union, 2013.

GALGANI, F.; HANKE, G. ; WERNER, S.; DE VREES, L. Marine litter within the european marine strategy framework directive. ICES Journal of Marine Science, $v$. $70, \quad$ n. $6, \quad$ p. 1055-1064, 2013. Disponível em: < http://dx.doi.org/10.1093/icesjms/fst122>. doi:10.1093/icesjms/fst122.

GIGAULT, J.; HALLE, A. T.; BAUDRIMONT, M.; PASCAL, P-Y.; GAUFFRE, F.; PHI, T-L.; HADRI, H. E.; GRASSL, B.; REYNAUD, S. Current opinion: What is a nonoplastic? Environmental Pollution, v. 235, p. 1-5, 2018. Disponível em:<https://doi.org/10.1016/j.envpol.2018.01.024>.

doi:10.1016/j.envpol.2018.01.024.

HATJE, V.; COSTA, M. F.; CUNHA, L. C. Oceanografia e química: unindo conhecimentos em prol dos oceanos e da sociedade. Química Nova, v. 36, n. 10, p. 1497-1508, 2013. Disponível em: <http://dx.doi.org/10.1590/S010040422013001000004>. doi:10.1590/S0100-40422013001000004.

HORTON, A. A.; WALTON, A.; SPURGEON, D. J.; LAHIVE, E.; SVENDSEN, C. Microplastics in freshwater and terrestrial environments: Evaluating the current understanding to identify the knowledge gaps and future research priorities. Science of the Total Environment, v. 586, p. 127-141, 2017. Disponível em: <https://doi.org/10.1016/j.scitotenv.2017.01.190>. doi:10.1016/j.scitotenv.2017.01.190.

HURLEY, R. R.; NIZZETTO, L. Fate and occurrence of micro(nano)plastics in soils: Knowledge gaps and possible risks. Current Opinion in Environmental Science \& 
Health, $\quad$ v. $1, \quad$ p. 6-11, 2018. Disponível em: <https://doi.org/10.1016/j.coesh.2017.10.006>. doi:10.1016/j.coesh.2017.10.006.

IMHOF, H. K.; LAFORSCH, C.; WIESHEU. A. C.; SCHIMID, J.; ANGER, P. M.; NIESSNER. R.; IVLEVA. N'. Pigments and plastic in limnetic ecosystems: a qualitative and quantitative study on microparticles of different size classes. Water Research, v. 98, p. 64-74, 2016. Disponível em: <https://doi.org/10.1016/j.watres.2016.03.015>. doi:10.1016/j.watres.2016.03.015.

JIANG, J. Q. Occurrence of microplastics and its pollution in the environment: a review. Sustainable Production and Consumption, v. 13, p. 16-23, 2018. Disponível em: <https://doi.org/10.1016/j.spc.2017.11.003>. doi: 10.1016/j.spc.2017.11.003.

KARAMI, A. GOLIESKARDI, A.; LARAT, V.; KARBALAEI, S.; SALAMATINIA, B. Microplastic and mesoplastic contamination in canned sardines and sprats. Science of The Total Environment, v. 612, p. 1380-1386, 2018. Disponível em: $<$ https://doi.org/10.1016/j.scitotenv.2017.09.005>.

doi:10.1016/j.scitotenv.2017.09.005.

KOELMANS, A. A. Modeling the role of microplastics in bioaccumulation of organic chemicals to marine aquatic organisms: A Critical Review . Marine Anthropogenic Litter, p. 313-328, 2015. Disponível em: < https://doi.org/10.1007/978-3-319-16510-3_11>. doi:10.1007/978-3-319-165103_11.

LAMBERT, S.; WAGNER, M. Characterisation of nanoplastics during the degradation of pollystyrene. Chemosphere, v. 145, p. 265-268, 2016. Disponível em: < http://doi.org/10.1016/j.chemosphere.2015.11.078>. doi:10.1016/j.chemosphere.2015.11.078.

MA, Y.; HUANG, A.; CAO, S.; SUN, F.; WANG, L.; GUO, H.; JI, R. Effects of nanoplastics and microplastics on toxicity, bioccumulation and environmental fate of phenantrhrene in fresh water. Environment Pollution, v. 2019. p. 166173, 2016. Disponível em: <https://doi.org/10.1016/j.envpol.2016.10.061>. doi:10.1016/j.envpol.2016.10.061.

MANO, E.B.; MENDES, L.C. Introdução a polímeros. $2^{\mathrm{a}}$ ed. São Paulo: Edgard Blucher, 1999. p. 3.

MARINE LITTER SOLUTIONS. Global Declaration. 2018. Disponível em: <https://www.marinelittersolutions.com/about-us/joint-declaration/>. Acesso em: 20 março 2018.

MENDOZA, L. M. R.; KARAPANAGIATI, H.; ÁLVAREZ, N. R. Micro(nanoplastics in the marine environment: curren knowledge and gaps. Corrent Opinion in Environmental Science e Health, v. 1, p. 47-51, 2018. Disponível em: <https://doi.org/10.1016/j.coesh.2017.11.004>. doi:10.1016/j.coesh.2017.11.004. 
MINISTÉRIO DA SAÚDE. Portaria $n^{\circ} 2.914$, de 12 de dezembro de 2011. Dispõe sobre os procedimentos de controle e de vigilância da qualidade da água para consumo humano e seu padrão de potabilidade. Diário Oficial da União, Poder Legilativo, Brasília, DF, 12 dez. 2011.

PAÇO, A., DUARTE, K.; COSTA, J. P.; SANTOS, P. S.. M.; PEREIRA, R.; PEREIRA, M. E.; FREITAS, NA. C. DUARTE, A. C.; ROCHA-SANTOS, T. A. P. Biodegradation of polyethylene microplastics by the marine fungus Zalerion maritimum. Science of The Total Environment, v. 586, p. 10-1515, 2017. Disponível em: <https://doi.org/10.1016/j.scitotenv.2017.02.017>. doi:10.1016/j.scitotenv.2017.02.017.

PLASTICSEUROPE - ASSOCIATION OF PLASTICS MANUFACTURES. Plastics the Facts 2017. Europe, 2017. Disponível em : $<$ http://www.plasticseurope.org/application/files/2415/1689/2630/2017plastics the fa cts.pdf>. Acesso em: 22 fev. 2018.

PRATA, J. C. Airborne microplastics: consequences to human health?. Environmental Pollution, v. 234, p. 115-126, 2018. Disponível em: < https://doi.org/10.1016/j.envpol.2017.11.043 0269-7491>. doi:10.1016/j.envpol.2017.11.043 0269-7491.

QIU, Q.; TAN, Z.; WANG, J.; PENG, J.; LI, M.; ZHAN, Z. Extraction, enumeration and identification methods for monitoring microplastics in the environment. Estuarine, Coastal and Shelf Science, v. 176, p. 102-109, 2016. Disponível em: <https://doi.org/10.1016/j.ecss.2016.04.012>. doi:10.1016/j.ecss.2016.04.012.

RAFIEE, M.; DARGAHI, L.; ESLAMI, A.; BEIRAMI, E.; JAHANGIRI-RAD, M.; SABOUR, S.; AMEREH, F. Neurobehavioral assessment of rats exposed to pristine polystyrene nanoplastics upon oral exposure. Chemosphere, v. 193, p. 745-753, 2018. Disponível em: <https://doi.org/10.1016/j.chemosphere.2017.11.076>. doi:10.1016/j.chemosphere.2017.11.076.

RENNER, G.; SCHMIDT, T. C.; SCHRAM, J. Analytical methodologies for monitoring micro (nano) plastics: Which are fit for purpose? Environmental Science \& Health, v. 1, p. 55-61, 2018. Disponível em: <https://doi.org/10.1016/j.coesh.2017.11.001>. doi:10.1016/j.coesh.2017.11.001.

REVEL, M.; CHÂTEL, A.; MOUNEYRAC, C. Micro(nano)plastics: a threat to human health? Current Opinion in Environmental Science e Health, v. 1, p. 17-23, 2018. Disponível em: <https://doi.org/10.1016/j.coesh.2017.10.003>. doi:10.1016/j.coesh.2017.10.003.

SCHYMANSKI, D.; GOLDBECK, C.; HUMPF, H-U.; FURST, P. Analysis of microplastics in water by micro-Raman spectroscopy: Release of plastic particles from different packaging into mineral water. Water Research, v. 129, p. 154-162, 2018. Disponível em: <https://doi.org/10.1016/j.watres.2017.11.011>. doi:10.1016/j.watres.2017.11.011. 
SCHIRINZI, G. F.; PÉREZ-POMEDAB, I.; SANCHÍSA, J. ROSSINIC, C.; FARRÉA, M.; BARCELÓA, D. Cytotoxic effects of commonly used nanomaterials and microplastics on cerebral and epithelial human cells. Environmental Research, v., p. 579-587, 2017. Disponível em: <https://doi: 10.1016/j.envres.2017.08.043> doi:10.1016/j.envres.2017.08.043.

SILVA, J. B.; BASTOS, A. S.; JUSTINO, C. I. L.; COSTA, J. P.; DUARTE, A. C.; ROCHA-SANTOS, T. A. P. Microplastics in the environment: Challenges in analytical chemistry - A review. Analytica Chimica Acta, v. 1017, p.1-19, 2018. Disponível em: < https://doi.org/10.1016/j.aca.2018.02.043>. doi:10.1016/j.aca.2018.02.043.

SJOLLEMA, S. B.; REDONDO-HASSELERHARM, P.; LESLIE, H. A.; KRAAK, M. H. S.; VETHAAK, A. D. Do plastic particles affect microalgal photosynthesis and growth?. Aquatic Toxicology, v. 170, p. 259-261, 2016. Disponível em: $<$ http://dx.doi.org/10.1016/j.aquatox.2015.12.002>.

doi:10.1016/j.aquatox.2015.12.002.

THOMPSON, R. C.; OLSEN, Y.; MITCHELL, R. P.; DAVIS, A. S.; ROWLAND, J.; JOHN, A. W. G.; MCGONIGLE, D.; RUSSELL, A. E. Lost at sea: where is all the plastic? Science, v. 304, n. 5672, p. 838, 2004. Disponível em: <https://doi: 10.1126/science.1094559>. doi:10.1126/science.1094559.

WAGNER, M.; SCHERER, C.; ALVAREZ-MUNOZ, D.; BRENNHOLT, N.; BOURRAIN, X.; BUCHINGER,S.; FRIES, E.; GROSBOIS, C.; KLASMEJER, J.; MARTI, T.; RODRIGUEZ-MOZAZ, S.; URBATZKA, R.; VETHAAK, A. D.; WINTHERNIELSEN, M.; REIFFERSCHEID,G. Microplastics in freshwater ecosystems: what we know and what we need to know. Environmental Sciences Europe, v. 26, n. 12, p. 1-9, 2014. Disponível em: <https://doi.org/10.1186/s12302-014-0012-7>. doi:10.1186/s12302-014-0012-7. 